\title{
Allergic Bronchopulmonary Aspergillosis: Diagnostic and Treatment Challenges
}

Lucia Leonardi*, Bianca Laura Cinicola, Rossella Laitano and Marzia Duse

Department of Pediatrics and Child Neuropsychiatry, Division of Allergy and Clinical Immunology, Sapienza University of Rome, Policlinico Umberto I, Rome, Italy

\begin{abstract}
Allergic bronchopulmonary aspergillosis (ABPA) is a pulmonary disorder, occurring mostly in asthmatic and cystic fibrosis patients, caused by an abnormal T-helper 2 lymphocyte response of the host to Aspergillus fumigatus antigens. ABPA diagnosis is defined by clinical, laboratory and radiological criteria including active asthma, immediate skin reactivity to $A$. fumigatus antigens, total serum $\mathrm{lgE}$ levels $>1000 \mathrm{IU} / \mathrm{mL}$, fleeting pulmonary parenchymal opacities and central bronchiectases that represent an irreversible complication of ABPA. Despite advances in our understanding of the role of the allergic response in the pathophysiology of ABPA, pathogenesis of the disease is still not completely clear. In addition, the absence of consensus regarding its prevalence, diagnostic criteria and staging limits the possibility of diagnosing the disease at early stages. This may delay the administration of a therapy that can potentially prevent permanent lung damage. Long-term management is still poorly studied. Present primary therapies, based on clinical experience, are not yet standardized. These consist in oral corticosteroids, which control acute symptoms by mitigating the allergic inflammatory response, azoles and, more recently, anti-lgE antibodies. The latter two are used as a steroid-sparing agent to prolong the remission stage of the disease. Anti-IgE antibodies also have immunomodulatory properties. At present, the only way to bypass these limits and allow for an early diagnosis, is to assume ABPA in all patients with difficult-to-control asthma or cystic fibrosis. They should then be screened for sensitization to $A$. fumigatus antigens and, if positive, monitored more closely. Future controlled studies are needed to standardize present therapy, standardize cut-off values of various investigations, define the role of different novel immunomodulatory therapies, define the role of novel assays (such as recombinant $A$. fumigatus antigens and CCL17) and confirm new diagnostic and staging criteria.
\end{abstract}

Keywords: ABPA; Asthma; Cystic fibrosis; Diagnosis; Therapy

\section{Introduction}

Allergic bronchopulmonary aspergillosis (ABPA) is an immunological pulmonary disease resulting from a hypersensitivity to Aspergillus antigens, more specifically to Aspergillus fumigatus (A. fumigatus). ABPA definition is sometimes improperly used to define hypersensitivity to other Aspergillus species. In that case the appropriate definition is allergic bronchopulmonary mycosis (ABPM) clinically identical to ABPA but caused by other fungi including Fusarium, Schizophyllum, Curvularia and Aspergillus species other than A. fumigatus [1]. First reported in detail in 1952 by Hinson et al. [2], ABPA occurs mainly as a complication in patients affected by asthma or cystic fibrosis (CF) who have been sensitized to A. fumigatus [1] (hereafter, asthma-ABPA and CF-ABPA patients). Coexisting atopy is often observed in these patients.

Rarely, ABPA occurs in patients with chronic granulomatous disease, hyperimmunoglobulinemia E, chronic eosinophilic pneumonia, Churg-Strauss syndrome, parasitic infestations, lymphoma and in lung transplant recipients [3,4]. An early diagnosis of ABPA, due to clinical, serological and radiological findings, is important in order to try to prevent lung damage resulting from inadequate or delayed therapy. This review is focused on challenges that caregivers can come across while diagnosing and treating this disease. A comprehensive search was conducted on December 2015 on MEDLINE literature via PubMed. Citations were identified on the electronic database using the terms "bronchopulmonary aspergillosis" or "ABPA" and "diagnosis" and "therapy" or "treatment". To reduce the risk of missing relevant studies, searches were not restricted by publication type or study design. Relevant reviews and clinical studies published on this topic were included as well, although not highlighted by our initial search.

\section{Epidemiology}

The lack of standardized diagnostic criteria for ABPA limits the estimation of prevalence of this condition, which remains speculative.
In addition, the disease is still misdiagnosed (mostly as pulmonary tuberculosis) in developing countries [5]. ABPA occurs almost exclusively in patients of both sexes with asthma or CF who have concomitant atopy and its diagnosis is based on clinical, biological and radiological criteria. The global prevalence of asthma-ABPA patients has been estimated to be as high as $2.5 \%$. Denning et al. [6] in addition, estimated the global burden of 4,837,000 ABPA patients in a worldwide asthma population.

The prevalence of CF-ABPA patients, according to a meta-analysis including 45 studies, conducted by Maturu and Agarwal [7], ranges from $3 \%$ to $25 \%$, with a mean value of $8.9 \%$, with a higher prevalence in adults in comparison with children. The wide range of prevalence is due to a heterogeneity in diagnostic criteria. Geller et al. [8] reported a slightly higher prevalence in male than in female patients with ABPA and CF. Other studies on CF-ABPA patients showed a lower agerelated prevalence in patients younger than 5-6 years and an increasing prevalence from ages 6 to 20 [9].

\section{Pathogenesis}

ABPA is one of many A. fumigatus related diseases and results from a hyperreactive immune response to the fungus without the tissue invasion that occurs in immunosuppressed patients (invasive

*Corresponding author: Lucia Leonardi, Department of Pediatrics, Division of Allergy and Clinical Immunology, Sapienza University of Rome, Policlinico Umberto I, Rome, Italy, Tel: +39 3471277845; Fax +39 0649979377; E-mail: lucialeonardi@yahoo.it, lucia.leonardi@uniroma1.it

Received June 21, 2016; Accepted July 15, 2016; Published July 19, 2016

Citation: Leonardi L, Cinicola BL, Laitano R, Duse M (2016) Allergic Bronchopulmonary Aspergillosis: Diagnostic and Treatment Challenges. J Pulm Respir Med 6: 361. doi: 1 0.4172/2161-105X.1000361

Copyright: (c) 2016 Leonardi L, et al. This is an open-access article distributed under the terms of the Creative Commons Attribution License, which permits unrestricted use, distribution, and reproduction in any medium, provided the original author and source are credited. 
pulmonary aspergillosis (IPA)). ABPA complex pathophysiology depends upon both the host's response and the characteristics of $A$. fumigatus. This saprophytic, spore-forming, and ubiquitous fungus is the most common type of outdoor and indoor environmental mold with spores that optimally grow at $15^{\circ} \mathrm{C}$ to $53^{\circ} \mathrm{C}[10]$. Spores are easily inhaled ( $2 \mu \mathrm{m}$ to $5 \mu \mathrm{m}$ in diameter) through the carina; they settle in distal and terminal airways, where they germinate in hyphae, especially in favorable environments such as mucus. Hyphae are able to release proteases that are responsible for the degradation of the epithelial cell layer [11]. As a result, the diffusion of antigens is promoted through the bronchial epithelium. Once recognized by antigen presenting cells, the latter activate the host immune response by releasing specific cytokines and by presenting A. fumigatus antigens to naive $\mathrm{T}$ lymphocytes, inducing an exaggerated T-helper class II (Th2) response (specific to ABPA) instead of a Th1 response. Notably, Th2 cell priming and impairment of macrophage activities are orchestrated by the Th2associated chemokine CCL17. In addition, Th2 cytokines (IL-4, IL-5, IL-13) stimulate B lymphocytes to produce specific A. fumigatus IgE that are responsible for type I hypersensitivity reaction in the airways mostly characterized by eosinophil recruitment that causes local inflammation.

However, only a small percentage of colonized subjects develops sensitization to the fungus and an even smaller subset of those sensitized patients progresses towards ABPA [12]. Thus, the development of the disease may depend more on the host characteristics, some of which are described below.

Above all, several genetic factors implicated in ABPA susceptibility have been identified. CF and asthmatic patients, who express HLADR2 and/or HLA-DR5 alleles, are at major risk of developing ABPA. By contrast, the HLA-DQ2 genotype seems to provide protection from ABPA [13]. In addition, ABPA susceptibility has been associated with several genetic polymorphisms [14]. Polymorphism of IL-4RA occurs in $95 \%$ of ABPA patients and promotes B cells IgE isotype switching [15]. IL-10 promoter polymorphisms promote an A. fumigatus specific Th2 response [16].

Single Nucleotide Polymorphisms (SNPs) in the collagen region of pulmonary surfactant protein A2 (SP-A2) have been correlated with higher IgE levels and higher percentages of eosinophilia in ABPA patients. The collagen region including these SNPs is likely associated with the coding region for receptors of alveolar macrophages implicated in host defense against $A$. fumigatus colonization [17].

Moreover, CFTR gene mutations detected in asthma-ABPA patients without CF might be implicated in abnormalities of mucus that promote trapping and proliferation of A. fumigatus spores [18]. In fact, Lebecque et al. [19] hypothesized that ABPA in pancreatic-sufficient adults is a CFTR-related disorder. As ABPA is often associated with bronchiectases (that also represent a phenotypic feature of $\mathrm{CF}$ ), further investigations (sweat test) are indicated to exclude milder forms of CF in these patients. Finally, among genetically-determined factors, atopy represents an important risk factor for ABPA. Other associated atopic conditions are highly represented in ABPA patients [20].

Among non-genetic host-related risk factors implicated in ABPA pathogenesis, the role of higher cumulative doses of inhaled corticosteroids (ICS) has been investigated. In a study conducted by Ritz et al. [21] on risk factors for ABPA in 160 CF patients, higher cumulative doses of ICS were significantly associated with $A$. fumigatus sensitization, but not with ABPA. Because ABPA, when occurring in asthmatic patients, affects mostly corticosteroid-dependent subjects, it would be useful to investigate the possible effect of cumulative doses of
ICS as a risk factor for asthma-ABPA patients as well. In fact, while ICS are an effective and safe therapy in asthma patients, at the low doses usually required, it is possible to hypothesize that higher doses of ICS can impair immune functions, most importantly macrophage killing, leading to increased germination of $A$. fumigatus [9,21].

Finally, new insights into ABPA pathogenesis have been supplied by recent investigations on pulmonary microbiome.

Several studies investigating the airways microenvironment of ABPA patients reported an association between detection of $A$. fumigatus and other microbes including Alternaria, Candida albicans, Pseudomonas aeruginosa and Burkholderia cepacia [9]. More specifically, the evidence of a coexistent colonization of $A$. fumigatus and $P$. aeruginosa in studies conducted on CF-ABPA patients allowed the postulate of a synergistic effect of these microbes on lung functions [12].

These results support the hypothesis that some bacterial or fungal microbes in the airway microenvironment can promote its colonization and sensitization by A. fumigatus. Although the involved microbe-tomicrobe interaction is still unclear, it is possible to suppose that damage of bronchial epithelium due to some pathogens can facilitate the colonization by secondary microbes.

\section{Diagnosis}

ABPA diagnosis presently relies on integrated clinical, radiographic, and serologic features (Table 1). There is no single specific and sensitive test or universally recognized criteria thus far.

\section{Clinical Features}

With the development of ABPA, asthma or CF typically worsens. ABPA onset generally presents with exacerbation symptoms resembling poorly controlled asthma (increased wheezing, increased coughing) as well as expectoration of brown mucous plugs and hemoptysis. These symptoms always require an evaluation for ABPA in a patient with asthma or CF [20,22].

In some cases, malaise, low-grade fever and weight loss are also observed [20], while patients with ABPA exacerbations are rarely asymptomatic and may be diagnosed solely on the basis of radiographic and/or serologic abnormalities [23].

Clinical presentation in CF-ABPA patients is extremely non-specific because it overlaps with the symptoms and signs of CF exacerbations. Viscosity of mucus, even more extreme than in CF, is one typical feature of ABPA exacerbations. In addition, the latter should be considered when the treatment of a suspected bacterial bronchial infection is ineffective [4].

Deterioration of lung function, bronchiectases and pulmonary fibrosis are considered the most fearsome complications for both asthma- and CF-ABPA patients. An unusual manifestation of CFABPA patients may be pneumomediastinum [24].

Findings during physical examination are usually similar to asthma, revealing wheezing and a prolonged expiratory phase. Clubbing, pulmonary hypertension and cyanosis may be present at advanced stages of the disease [11].

\section{Diagnostic criteria for asthma-ABPA patients}

Several diagnostic criteria for asthma-ABPA patients have been suggested over the past several decades (Table 2). In 1977, Rosenberg et al. [25] formulated diagnostic criteria, still widely accepted, that included seven relevant findings defined as "primary criteria": asthma, 
Citation: Leonardi L, Cinicola BL, Laitano R, Duse M (2016) Allergic Bronchopulmonary Aspergillosis: Diagnostic and Treatment Challenges. J Pulm Respir Med 6: 361. doi: 10.4172/2161-105X.1000361

Page 3 of 11

\begin{tabular}{|c|c|c|}
\hline Findings & CF & Asthma \\
\hline \multicolumn{3}{|c|}{ Clinical Features } \\
\hline & Worsening wheezing & Worsening wheezing \\
\hline & $\begin{array}{l}\text { Increase in productive cough with expectoration of brown } \\
\text { mucous plugs }\end{array}$ & $\begin{array}{l}\text { Increase in productive cough with expectoration of brown mucous } \\
\text { plugs }\end{array}$ \\
\hline & Hemoptysis & Hemoptysis \\
\hline & Malaise & Malaise \\
\hline & Low-grade fever & Low-grade fever \\
\hline & Weight loss & Weight loss \\
\hline \multicolumn{3}{|c|}{ Serological Findings } \\
\hline Skin prick test & $>3 \mathrm{~mm}$ & $>3 \mathrm{~mm}$ \\
\hline Total serum IgE levels & $\begin{array}{l}>500 \mathrm{IU} / \mathrm{mL} \text { (minimal criteria) } \\
>1000 \mathrm{IU} / \mathrm{mL} \text { (classic criteria) }\end{array}$ & $>1000 \mathrm{IU} / \mathrm{mL}$ \\
\hline Serum $\lg E$ to $A$. fumigatus & $>0.35 \mathrm{KU} / \mathrm{L}$ & $>0.35 \mathrm{KU} / \mathrm{L}$ \\
\hline Eosinophilia & not considered & $>500$ cells $/ \mu \mathrm{L}$ \\
\hline $\begin{array}{l}\text { Precipitins and Serum IgG to } A \text {. } \\
\text { fumigatust }\end{array}$ & not significant & not significant \\
\hline Recombinant Aspergillus Ag & promising & data not available \\
\hline BAT (basophil activation test) & promising & data not available \\
\hline CCL17 (serum) & promising & data not available \\
\hline Galactomannan (serum) & not significant & not significant \\
\hline \multicolumn{3}{|c|}{ Radiological Findings } \\
\hline & Fleeting parenchymal opacities in upper lobes & Fleeting parenchymal opacities in upper lobes \\
\hline & Central bronchiectases & Central bronchiectases \\
\hline & Mucoid impaction & Mucoid impaction \\
\hline & Bronchial wall thickening & Bronchial wall thickening \\
\hline & Atelectasis & Atelectasis \\
\hline & Lobar or whole lung collapse & Lobar or whole lung collapse \\
\hline & End-stage findings: Fibrosis and cavitations & End-stage findings: Fibrosis and cavitations \\
\hline
\end{tabular}

Table 1: Diagnostic findings in CF-ABPA and Asthma-ABPA.

\begin{tabular}{|c|c|c|}
\hline Rosenberg et al. [25] & Schwartz and Greenberger [28] & Agarwal et al. [23] \\
\hline Primary criteria: & ABPA-CB: & Obligatory criteria: \\
\hline \multirow{4}{*}{$\begin{array}{l}\text { - Asthma } \\
\text { - Elevated total serum IgE levels } \\
\text { - Immediate skin reactivity to A. fumigatus } \\
\text { - Serum eosinophilia } \\
\text { - Precipitins } \\
\text { - Central bronchiectases } \\
\text { - History of pulmonary infiltrates } \\
\end{array}$} & \multirow{3}{*}{$\begin{array}{l}\text { - Asthma } \\
\text { - Positive skin test to A. fumigatus } \\
\text { - Elevated Total serum IgE levels } \\
\text { - Serum IgE and IgG to A. fumigatus } \\
\text { - Central bronchiectases }\end{array}$} & \multirow{8}{*}{$\begin{array}{l}\text { - Positive skin test and/or serum IgE to } A \text {. } \\
\text { fumigatus } \\
\text { - } \text { Elevated total serum IgE levels } \\
\text { Other criteria }(\geq 2) \text { : } \\
\text { - Precipitins or serum IgG to A. fumigatus } \\
\text { - Serum eosinophilia } \\
\text { - Radiological pulmonary findings consistent with } \\
\text { ABPA }\end{array}$} \\
\hline & & \\
\hline & & \\
\hline & - Asthma & \\
\hline Secondary criteria: & - Positive skin test to A. fumigatus & \\
\hline \multirow{3}{*}{$\begin{array}{l}\text { - A. fumigatus in sputum } \\
\text { - History of brown plugs or flecks expectoration } \\
\text { - Late skin reactivity to } A \text {. fumigatus }\end{array}$} & $\begin{array}{l}\text { - Elevated Total serum IgE levels } \\
\text { - Serum IgE and IgG to A. fumigatus }\end{array}$ & \\
\hline & Additional findings: & \\
\hline & $\begin{array}{l}\text { - Mucus plugs } \\
\text { - A. fumigatus in sputum } \\
\text { - Precipitins } \\
\text { - Parenchymal infiltrates } \\
\text { - Delayed positive skin test }\end{array}$ & \\
\hline
\end{tabular}

Table 2: Diagnostic criteria for asthma-ABPA patients.

parenchymal infiltrates, elevated total serum IgE levels, cutaneous reactivity to $A$. fumigatus, serum eosinophilia, precipitating antibodies against A. fumigatus and central/proximal bronchiectasis. A few years later, eosinophilia and pulmonary infiltrates ceased to be considered essential for ABPA diagnosis because these findings can be present only during exacerbations [26].

In 1986, Patterson et al. [27] highlighted that bronchiectases, considered a major criterion for ABPA diagnosis, can be absent in early stage ABPA. Therefore, in 1991, diagnostic criterion was revised by classifying ABPA patients into two categories: ABPA-CB (ABPA with central bronchiectases) and ABPA-S (ABPA seropositive without bronchiectases) [28]. The frequency of exacerbations and the probability of permanent lung damage are believed to be lower in patients with ABPA-S compared to ABPA-CB patients [20].

Agarwal et al. [23] proposed a new set of diagnostic criteria with the aim of overcoming the traditional classification and suggesting an optimum cut-off value for total serum IgE levels while not considering bronchiectases, which can develop much later in ABPA natural history. Agarwal et al. [23] established that ABPA diagnosis should first be formulated by the presence of two obligatory criteria - positivity of skin prick test and/or elevated serum IgE to A. fumigatus and total serum IgE levels $>1000 \mathrm{IU} / \mathrm{mL}$ and at least two of three "other criteria" including precipitins or IgG antibodies to A. fumigatus, radiological 
pulmonary findings consistent with ABPA, and total eosinophil count $>500$ cells $/ \mu \mathrm{L}$ (in patients not treated with steroids) [23].

\section{Diagnostic criteria for CF-ABPA patients}

Diagnosis in CF-ABPA patients may be particularly challenging because typical clinical and radiological ABPA findings are often present at baseline in CF patients. A high level of suspicion for ABPA should be kept in CF patients older than 6 years of age. The diagnostic criteria for CF-ABPA patients are reviewed extensively and most recently in the $\mathrm{CF}$ Foundation Consensus Conference (CFFCC) statement where ABPA is suggested in case of clinical deterioration (worsening cough, exercise intolerance or wheezing, increased sputum and decline in pulmonary function test, not attributable to another etiology). This should lead to skin testing for A. fumigatus (a wheal $>3 \mathrm{~mm}$ in diameter is considered positive) and measurement of total serum IgE levels (suggestive of ABPA if $>1000 \mathrm{IU} / \mathrm{mL}$ for patients not treated with systemic corticosteroids).

In addition, new or recent radiological findings, at chest imaging, not responsive to standard treatment (antibiotics and physiotherapy) can help distinguish infiltrates due to ABPA over an active infectious process. Total serum IgE levels $>500 \mathrm{IU} / \mathrm{mL}(1200 \mathrm{ng} / \mathrm{mL})$ are considered a minimal diagnostic criterion for CF-ABPA patients (Table 3). In CF patients with highly suspected ABPA and total serum IgE value of $200 \mathrm{IU} / \mathrm{mL}$ to $500 \mathrm{IU} / \mathrm{mL}$, it is advised to reevaluate IgE levels in 1-3 months [22,24].

\section{Clinical Staging of ABPA}

ABPA may be progressive. Staging of the disease was first proposed by Rosenberg et al. [25] and has been mostly applied in the clinical and therapeutic follow-up of asthma-ABPA patients in order to discriminate active disease from remission or end-stage bronchiectases, the latter leading to progressive lung damage.

Patterson et al. [26] classified ABPA into five stages: acute stage I describes patients with elevated total serum IgE levels, eosinophilia and infiltrates at chest radiographs; stage II is characterized by the absence of radiological findings after discontinuation of oral steroids for more than 6 months (remission); recurrent exacerbations define stage III; steroid dependent patients are classified in stage IV; stage V defines fibrotic disease. Progression from one stage to another is not necessarily sequential.

Agarwal et al. [23] proposed a new clinical staging of asthmaABPA by introducing a stage 0 for asymptomatic patients diagnosed with $A B P A$ after routine investigation, while stage 1 is referred to acute ABPA. Stage 2 is described as a "response stage" that includes improvement in symptoms, clearing of radiographic opacities and at least a $25 \%$ decline in total serum IgE levels (generally occurring after 8 weeks of therapy). Stage 3, occurring in $25 \%$ to $50 \%$ of ABPA patients, is characterized by an increase of $50 \%$ in baseline total serum IgE levels and associated with clinic-radiological worsening. Baseline total serum IgE levels generally refers to the earliest IgE test available or is obtainable in the absence of radiological findings during remission (stage 4), which is defined by the absence of exacerbations over 6 months. Patients in stage 4 require a follow-up with a measurement of total serum IgE levels every 3-6 months. Patients with difficult to control ABPA symptoms are identified by stage 5 , while stage 6 includes patients with widespread bronchiectases or fibrosis [23].

\section{Diagnostic Test Findings}

\section{Skin testing}

In patients with suspected ABPA, skin testing for A. fumigatus followed by total serum IgE levels measurement should always be performed. A positive Skin Prick Test (SPT) for A. fumigatus is due to IgE-mediated response (hypersensitivity) to A. fumigatus antigens and it is considered the gold standard screening for ABPA. SPT is highly sensitive for A. fumigatus IgE mediated response (90\%) but is not specific to ABPA: up to $40 \%$ of patients without ABPA can demonstrate a sensitization to A. fumigatus antigens [29]. In addition, some patients may only show hypersensitivity with an intradermal test, which should be performed only in case of a negative SPT [23].

\section{Serologic assays}

Confirmatory diagnostic serologic assays can be performed in the event of a positive SPT. More specifically, measurement of total serum IgE levels is useful in both diagnosis and follow up of ABPA.

\section{Total serum IgE levels}

Active ABPA is generally excluded in corticosteroid-naïve patients with normal total serum IgE levels despite there is no consensus on the cut-off value that should be used for the diagnosis of the disease.

In addition, many studies report total serum IgE levels using different units, generating difficulties for the interpretation of data. Finally, the wide variation in IgE concentration in normal population represents an additional challenge in establishing precise cut-off values. Despite these limits, a cut-off value $>1000 \mathrm{IU} / \mathrm{mL}(>2400 \mathrm{ng} / \mathrm{mL})$ for asthma-ABPA patients has been suggested and employed thereafter in several studies [30].

For CF patients the total serum IgE levels suggested by the CFFCC that discriminate ABPA patients from sensitized patients is $>500 \mathrm{IU} /$ $\mathrm{mL}$ (defined as minimal diagnostic criteria for ABPA) with a sensitivity of $70 \%$ and specificity of $99 \%$. A level $>1000 \mathrm{IU} / \mathrm{mL}$ (defined as classic diagnostic criteria for ABPA) has a sensitivity of $39 \%$ and a specificity of $100 \%$ [22,23].

Total serum IgE levels are high at diagnosis or during exacerbations, but they decrease in the event of remission, glucocorticoids treatment and end-stage ABPA. Therefore, diagnosis should be based on the earliest IgE test available.

\section{IgE antibodies specific to $A$. fumigatus}

An elevated level of IgE antibodies specific to A. fumigatus is considered a characteristic finding of ABPA. A suggested value $>0.35$ $\mathrm{kU} / \mathrm{L}$ should be used as cut-off in discriminating ABPA patients from asthmatic patients sensitized to A. fumigatus [31].

\section{Cystic Fibrosis Foundation Consensus Conference [22]}

- Clinical deterioration (worsening cough, exercise intolerance or wheezing, increased sputum and decline in pulmonary function test, not attributable to another etiology).

- Positive skin test for $A$. fumigatus (a wheal $>3 \mathrm{~mm}$ in diameter) or serum $\lg E$ to $A$. fumigatus

- Total serum IgE levels (classic criteria: $>1000 \mathrm{IU} / \mathrm{mL}$; minimal criteria: $>500 \mathrm{IU} / \mathrm{ml}$ )

- Precipitins or serum IgG antibodies to $A$. fumigatus

- New or recent radiological findings, at chest imaging, not responsive to standard treatment (antibiotics and physiotherapy) 


\section{Serum eosinophilia}

Serum eosinophilia is not sensitive or specific, but if present, supports a diagnosis of ABPA.

\section{Serum precipitins or specific IgG against $A$. fumigatus}

Serum precipitins or specific IgG against A. fumigatus are not commonly used for ABPA diagnosis, because of a lack of standardized laboratory testing [20]. In addition, serum IgG against $A$. fumigatus may not be a specific test for ABPA diagnosis, as high levels are detectable in other forms of aspergillosis [23].

\section{Recombinant Aspergillus antigens}

The promising role of recombinant Aspergillus antigens in the detection of sensitization to A. fumigatus and in the diagnosis of ABPA has been recently recognised. Precisely, specific IgE against the recombinant allergens Asp f4 (rAsp f4) and/or Asp f6 (rAsp F6) were exclusively detected in the serum of individuals with ABPA allowing to consider them as specific markers of the disease. While antibodies against rAsp f1 and rAsp f3 are elevated in both subjects sensitized to A. fumigatus and in ABPA patients. Further studies are required to confirm the diagnostic values of these allergens $[32,33]$.

\section{Basophil activation test}

Basophil Activation Test (BAT) is a flow cytometry method that assess hyper-expression of CD203c on activated basophils in response to A. fumigatus. The assay has been proposed as a diagnostic alternative to identify $A$. fumigatus-sensitized CF patients. BAT can better discriminate between nonsensitized and A. fumigatus-sensitized $\mathrm{CF}$ patients when compared to A. fumigatus-IgE specific detection (A. fumigatus sensitization cut off $>0.35 \mathrm{kU} / \mathrm{L}$ ) [34].

\section{CCL17}

Serum levels of CCL17 (also known as TARC) are elevated in CF patients with ABPA. CCL17 is a chemokine produced as a result of the antifungal immune response. Recently, elevated levels of CCL17 have been confirmed as a sensitive and specific marker of ABPA in CF patients with diagnostic accuracy greater than total serum IgE and recombinant $A$. fumigatus antigens $\mathrm{f} 4$ and $\mathrm{f} 6$. In a study conducted by Latzin et al. [35], CCL17 levels were elevated in CF-ABPA patients even before the development of clinical features of ABPA and before total serum IgE elevation. The same authors in another study observed significantly higher serum levels of CCL17 in asthma-ABPA and CF-ABPA patients when compared to CF patients colonized with or sensitized to A. fumigatus [36]. Although the overall results from these studies are promising, this new marker requires further investigation in order to confirm its clinical potential for early detection of ABPA and for disease monitoring.

\section{Galactomannan}

Galactomannan (GM) is a polysaccharide component of the $A$. fumigatus cell wall. Testing this antigen with Platelia Aspergillus enzyme Immunoessay is the gold standard in the diagnosis of IPA.

Its role in the diagnosis of ABPA needs to be confirmed. In a recent study, GM has been tested in the serum of 70 asthma-ABPA patients compared with 50 asthmatic patients without ABPA; it has been observed that levels of serum GM were not significantly different between the two groups. The authors hypothesized that in immunocompromised patients with IPA it is possible to detect GM in serum because $A$. fumigatus manages to invade blood vessels, while in ABPA the integrity of the immune system prevents fungal dissemination [37]. GM could otherwise be detected in the bronchoalveolar fluid and in sputum of patients with ABPA.

\section{Radiological Findings}

Chest radiographs and Computerized Tomography (CT) are the diagnostic tools most widely used for ABPA. The most common findings in ABPA are fleeting parenchymal opacities and bronchiectases.

At chest X-Rays, ABPA eosinophilic opacities usually appear as a large, homogeneous shadow, more frequently in upper lobes that partially or completely disappear after steroid treatment, allowing to differentiate from infectious pneumonia.

Mucoid impaction may not be seen at chest radiographs but exclusively on high resolution chest CT, considered the most sensitive radiological investigation in detecting the full spectrum of ABPA findings [38].

Mucoid impaction is characteristic of ABPA exacerbations and can be observed as small nodules or as a parallel line spreading from the hilum with a rounded distal margin (glove-finger shadow) when it opacifies an airway and its branches [39]. Hypodense mucus plugs are more commonly described in ABPA, but high-attenuation mucus, which suggests chronicity, is a pathognomonic finding of the disease, despite being observed in only $20 \%$ of patients [40].

Central bronchiectases (CB) are relevant findings that, in association with fibrosis, are considered irreversible alterations of pulmonary parenchyma. When observed on a chest radiography in an en face orientation, they appear like "ring shadows."

Bronchiectases in ABPA sometimes can be seen also in the peripheral lung fields. Although $\mathrm{CB}$ of upper lobes are a hallmark of ABPA, diagnosis can be made even in their absence because they represent a late feature of the disease [27].

Cylindrical, varicose and cystic bronchiectases (the latter two specific to ABPA) typically involve multiple bronchi. Their detection in three or more lobes is a highly suggestive and helpful clue in differentiating asthma and CF without ABPA, where bronchiectases are limited to one or two lobes, from asthma-and CF-ABPA [10].

Other radiological findings with CT include bronchial wall thickening, atelectasis, lobar or whole lung collapse. Fibrosis and cavitations of dilated airways are typically end-stage findings of the disease [41].

\section{Pulmonary Function Testing}

Because ABPA usually presents in asthmatic and CF patients, findings of pulmonary function test are not a sufficient indicator of the disease, but they are important to the follow up of ABPA-patients. Mild and early ABPA are commonly characterized by a partially reversible airflow obstruction, while fixed airflow obstruction reflects progressive remodeling of end stage disease [20].

\section{Bronchoscopy}

Histo-cytological sampling and fungal culture obtained with bronchoscopy do not provide findings considered among the diagnostic criteria for ABPA. However, bronchofiberscopy appears to be a useful tool in detecting ABPA in patients whose serologic and radiographic findings are not typical of ABPA [42]. In these cases, bronchoalveolar lavage can be useful to exclude alternate etiologies by showing elevated eosinophil counts, elevated IgE levels and A. fumigatus hyphae, while lung biopsy may typically show infiltration of airways by eosinophils 
and lymphocytes, mucoid impaction, and fibrotic changes in end-stage disease $[20,43,44]$. In addition, the pathological analysis of mucus plugs, obtained in ABPA patients during bronchofiberscopy by suction, is commonly characterized by clusters of eosinophils containing fungal hyphae in layers. On the contrary, bronchoalveolar lavage washes and sputum samples do not offer a reliable culture of $A$. fumigatus, even during exacerbations, while the recovery of $A$. fumigatus in culture may indicate only colonization and is not specific to active disease $[44,45]$. Fiberoptic bronchoscopy may finally have an important therapeutic role in removing the mucoid impaction (responsible for atelectasis, recurrent exacerbations and proximal collapse) in cases not responsive to steroid treatment [4].

\section{Differential diagnosis}

The differential diagnosis for ABPA includes ABPM, refractory asthma, newly diagnosed CF, infectious pneumonia (especially in CF patients), Churg-Strauss syndrome, aspergillus sensitive asthma, eosinophilic pneumonia, tuberculosis (most of all in endemic areas), bronchocentric granulomatosis and sarcoidosis $[20,30]$.

\section{Therapy}

Early diagnosis of ABPA is necessary to initiate prompt treatment with the aim of controlling symptoms, preventing exacerbations, inducing remission, and potentially preventing significant airway and parenchymal destruction.

Management of ABPA typically consists of systemic glucocorticoids, administered to attenuate the inflammatory response, and antifungal agents, which reduce the fungal load in the airways. Despite that, long-term outcomes remain poorly studied. Only a small number of randomized controlled trials have been conducted, which did not collect useful data on whether or not drugs typically administered slow the progression of ABPA to fibrotic lung disease.

\section{Systemic glucocorticoid therapy}

Oral corticosteroids in ABPA: Oral corticosteroids (OCS) are currently the mainstay of therapy for ABPA. Their anti-inflammatory action suppresses the immune hyper-reactivity of ABPA in both asthmatic and CF patients. Therapy with OCS leads to an improvement of symptoms and resolution of pulmonary infiltrates, and decreases total serum IgE levels and peripheral eosinophilia [11], but at considerable cost in terms of side effects, if chronically administered, such as gastric ulcers, osteoporosis, diabetes, and immune, adrenal and growth suppression.

OCS in asthma-ABPA patients: Although several case reports and case series supported OCS efficacy in asthma-ABPA patients, there are no standardized therapeutic schemes on doses and duration of therapy [46]. Two regimens for oral glucocorticoid therapy have been suggested in acute ABPA. Greenberger proposed a low-dose regimen of daily prednisone $0.5 \mathrm{mg} / \mathrm{kg} /$ day for $1-2$ weeks, followed by 6 to 8 weeks of alternate day therapy to be then tapered by $5 \mathrm{mg}$ to $10 \mathrm{mg}$ every 2 weeks up to discontinuation based on the individual clinical course [10]. Instead, Agarwal et al. [47] proposed a more aggressive approach with prednisolone $0.75 \mathrm{mg} / \mathrm{kg} / \mathrm{day}$ for 6 weeks, then $0.5 \mathrm{mg} /$ $\mathrm{kg} /$ day for 6 weeks, then tapered by $5 \mathrm{mg}$ every 6 weeks to continue for a total duration of at least 6-12 months [31]. In order to evaluate if higher doses or prolonged therapy with OCS are associated with better outcomes, a randomized controlled trial, comparing the two regimens by using only prednisolone, has been recently completed. It reported that the low dose in OCS is as effective and safer than the high dose in treatment of ABPA patients with asthma [47].
OCS in CF-ABPA patients: Therapy for ABPA is challenging in patients with $\mathrm{CF}$ because both conditions share clinical and physiological features [48]. Treatment of CF-ABPA patients is similar to asthma-ABPA patients, although higher doses of prednisone are recommended.

During CFFCC, guidelines on the treatment of ABPA exacerbations in CF patients were issued, suggesting an initial dose treatment of prednisone of $0.5 \mathrm{mg} / \mathrm{kg} /$ day to $2 \mathrm{mg} / \mathrm{kg} /$ day, maximum dose $60 \mathrm{mg}$, for $1-2$ weeks, then reduced to $0.5 \mathrm{mg} / \mathrm{kg} /$ day to $2 \mathrm{mg} / \mathrm{kg} /$ day on alternate days for 1-2 weeks. A clinical response confirms the diagnosis of ABPA exacerbation. Prednisone dose should then gradually be tapered within 2-3 months on the basis of clinical, radiological and serological findings. In case of relapse, it is suggested to increase OCS or to add antifungal therapy [22].

Systemic glucocorticoids are responsible for adverse effects, particularly in CF patients who are already at risk of developing osteopenia and infections. In long-term patients, vaccination for pneumococcus and influenza is advised and integration of calcium and Vitamin D should be considered [20]. Therapy must also take into account that the pharmacology of many drugs, such as enteric-coated prednisolone, is altered in CF patients due to an incomplete absorption [22]. Cases of IPA have been reported as a complication of long-term corticosteroid therapy in both asthma- and CF-ABPA [49].

In addition, studies show that many patients will require lifelong low-dose corticosteroid therapy to control symptoms. Almost 50\% of patients, after tapering OCS, relapse and $20 \%$ to $45 \%$ remain glucocorticoid-dependent [50]. Therefore, alternative approaches such as intravenous or inhaled corticosteroids, azoles or anti-IgE antibodies therapy have been developed for the management of ABPA.

\section{Intravenous corticosteroids}

Corticosteroid dependence is a state of steroid resistance due to down-regulation of steroid receptors in long-term steroid therapy. The pulse therapy of high doses of intravenous methylprednisolone (HDIVPM) may overcome this effect by limiting the degree of downregulation of the receptor and enhancing the clinical efficacy compared to daily oral steroids [51].

Pulse steroids therapy efficacy and safety has been demonstrated in two asthma-ABPA patients whose exacerbations were unresponsive to conventional therapy with OCS and antifungals. Pulse therapy with 1 $\mathrm{g} /$ day of intravenous methylprednisolone for 3 days was able to induce clinical stabilization and reduce the dose of OCS therapy within 4-8 weeks [52]. Efficacy of HDIVPM in the management of CF-ABPA patients has been described by Cohen-Cymberknoh et al. [53] in nine CF-ABPA patients treated with pulses of HDIVPM of $10 \mathrm{mg} / \mathrm{kg} /$ day to $15 \mathrm{mg} / \mathrm{kg} /$ day (max dose $1 \mathrm{~g} /$ day) for 3 consecutive days, once a month. HDIVPM was associated with antifungal therapy. An improvement in respiratory symptoms and pulmonary function and a reduction in total serum IgE levels were obtained after the second course of intravenous methylprednisolone. HDIVPM was discontinued after 6 to 10 pulses, while antifungal therapy continued from 8 months to over 5 years. Only mild and transient adverse events were reported.

\section{Inhaled corticosteroids}

The use of ICS in controlling ABPA symptoms or preventing pulmonary fibrosis is controversial. CFFCC remarked that extremely limited information is available on the efficacy of inhaled corticosteroids in CF-ABPA patients. Therefore, these drugs should not be recommended for initial therapy or for preventing pulmonary 
fibrosis [22]. In a recent study, 21 glucocorticoid-naïve asthmatic patients with ABPA-S have been treated with a combination of formoterol/budesonide $(24 / 1600 \mu \mathrm{g} /$ day $)$ with improvement of subjective symptoms in all patients treated.

Conversely, no patients gained complete control over their asthma, while the total serum IgE levels progressively increased in all patients over 6 months. Therefore, the authors concluded that ICS can be effective in controlling asthma symptoms, while are unable to control immunological activity in ABPA [49].

\section{Azoles}

Azoles are antifungal agents that have been reported to be a valid added therapy to long-term oral steroids in ABPA. Their action against airway fungal colonization can reduce antigenic stimulation and, consequently, inflammatory response, bringing an improvement in symptoms and lung function. Current recommendations suggest azoles therapy, combined with OCS, in steroid-dependent patients, in patients with recurrent exacerbations, or when corticosteroids alone are ineffective in controlling acute symptoms [22,23]. Generally, azoles combined with OCS have a steroid-sparing effect that allows the reduction of the corticosteroid dose and increases the interval between corticosteroid courses [54,55]. A number of different antifungal strategies have been tried in ABPA patients.

\section{Nebulized azoles}

Inhaled natamycin (5 mg twice daily for one year) has been tested in a controlled trial conducted on asthma-ABPA patients by Currie et al. [56] without proved steroid sparing effect. Better results on steroid sparing effect have been described in few case series and case reports on CF-ABPA patients with nebulized amphotericin B whose beneficial effects need to be confirmed in larger trials [57].

\section{Systemic azoles}

Oral ketaconazole has been used in ABPA [58] but has been replaced by itraconazole because of higher efficacy and lower side effects of the latter. The dose of azoles in ABPA has never been standardized, although a dose of $200 \mathrm{mg}$ twice a day of itraconazole po for recurrent 16-week courses or long-term therapy have been correlated to clinical efficacy [23].

Azoles in asthma-ABPA: In corticosteroid-dependent asthmaABPA patients, the effectiveness of itraconazole combined with OCS has been demonstrated during a randomized double-blind placebocontrolled trial using $200 \mathrm{mg}$ of itraconazole po twice daily for 16 weeks. The efficacy of itraconazole was confirmed by a reduction of at least $50 \%$ in the oral steroid dose, a decrease of at least $25 \%$ in total serum IgE levels, and an increase of at least $25 \%$ in exercise tolerance. In addition, there was an increase in at least one of five pulmonary function tests or resolution of pulmonary infiltrates in patients treated with itraconazole when compared to the placebo group. Minimal adverse effects were reported both in the itraconazole group and in the placebo one [59]. In order to analyze the anti-inflammatory effect of itraconazole on asthmatic patients with stable ABPA, Wark et al. [60] conducted a randomized double-blind placebo-controlled trial using $400 \mathrm{mg}$ of itraconazole po per day for 16 weeks. Patients treated with itraconazole showed a reduction in sputum eosinophils, sputum eosinophil cationic protein levels and in serum IgE and IgG levels to $A$. fumigatus compared to the placebo group. Itraconazole was also able to reduce the number of exacerbations requiring oral corticosteroids without adverse effects. These data suggest using itraconazole in asthmaABPA patients in order to prevent new exacerbations, after controlling them with glucocorticoid therapy. Agarwal et al. [23] highlighted that neither study reported outcomes for more than 8 months with regard to relapses of ABPA. Therefore, the long-term benefits of azole therapy remain unknown.

Azoles in CF-ABPA: There are no randomized controlled trials evaluating the use of antifungal therapies for the treatment of CFABPA patients [61]. The CFFCC recommends a dose of $5 \mathrm{mg} / \mathrm{kg} /$ day of itraconazole po, maximum dose $400 \mathrm{mg} / \mathrm{day}$, for 3-6 months combined with OCS in patients who have a poor response to corticosteroids, who are corticosteroid-dependent, or where there is evidence of corticosteroid toxicity. Liver function should be tested before the therapy, after the first month of antifungal therapy and then every 3-6 months [22].

In both asthma- and CF-ABPA patients, itraconazole serum levels (drawn 4 hours after a dose, following at least 1 week of therapy) [22] should be monitored to ensure adequate bioavailability and avoid toxicity. This therapeutic drug monitoring (TDM) is particularly important in patients who do not respond to itraconazole therapy. It is also recommended in patients who suffer from liver failure or malabsorption, or in subjects taking medication that reduces acid production [22]. The maintenance of an itraconazole therapeutic range could prevent clinical failure and the development of azole resistance, although no clear correlation has been established between drug levels and efficacy [23]. However, therapeutic drug monitoring (TDM) of itraconazole is limited because its serum concentration is influenced by dose-dependent pharmacokinetic [62], drug formulations (capsule, oral or intravenous solution) and by individual variations of cytochrome P450 activity [63].

Itraconazole minimal steady-state level can be measured by highperformance liquid chromatography (HPLC) or bioassay. When measured by HPLC, the trough level suggested is $0.5 \mathrm{mg} / \mathrm{l}$. The bioassay, detecting simultaneously itraconazole and its active metabolite, estimates itraconazole levels 2-10 times higher than those obtained by HPLC [12,64].

In addition, concomitant therapy should be considered in evaluating potential drug-drug interaction between itraconazole and several corticosteroids (intravenous methylprednisolone, inhaled budesonide or fluticasone) [65]. Itraconazole may increase the activity of corticosteroids by inhibiting their metabolism. As a result, onset of complications may occur including abnormal adrenocorticotropic hormone stimulation and profound immunosuppression leading to IPA [66]. Further studies are needed to investigate the possible efficacy of itraconazole monotherapy.

Other azoles: Voriconazole and posaconazole are newer azoles whose efficacy in asthma- and CF-ABPA patients has been demonstrated in some case series $[67,68]$. Despite that, placebo controlled studies have not yet been performed. In addition, these drugs showed a higher toxicity compared to itraconazole due to their stronger inhibitory effect on hepatic cytochrome P450 enzymes [69]. Serious side effects have been described with long-term voriconazole therapy, including skin cancer due to photosensitivity, induced by this drug, especially in phototypes II-III patients. For these reasons, itraconazole should be the first choice for antifungal therapy in ABPA while voriconazole or posaconazole should be reserved for patients with poor response or experiencing adverse effects to itraconazole.

\section{Patient Monitoring}

Follow up in treated patients with new ABPA diagnosis consists in performing total serum IgE levels, chest imaging and lung function 
test every 6-8 weeks for 1-2 years after the initiation of oral steroids, in order to evaluate response to therapy and to identify a possible relapse [70]. Successively, total serum IgE levels should be measured annually. Clinical effectiveness of therapy is reflected by a decreased of $25 \%$ to $50 \%$ of total serum IgE levels associated to clinical and radiological improvement [23]. Since an increase in total serum IgE levels may correlate to ABPA exacerbations, it is useful to obtain, while patients are in remission stage, a baseline serum level of total IgE to be monitored during follow up. Some authors have suggested starting corticosteroid therapy even for asymptomatic patients if the total serum IgE levels double from the baseline value [27]. However, although even an increase by $50 \%$ over baseline suggests recurrent disease [71], the decision of starting OCS therapy should also take into consideration other clinical and radiological parameters. Thus, ABPA therapy poses challenging issues. Up to date, no data confirm that combined therapy with OCS and azoles can prevent long-term complications. In addition, it is not clear if asymptomatic patients can benefit from any therapy. Finally, while azoles are effective in preventing ABPA recurrent exacerbations, their role in managing acute exacerbations is not clear.

\section{Other Therapeutic Aspects}

\section{Omalizumab}

Omalizumab is a humanized monoclonal antibody against $\operatorname{IgE}$ currently used to treat severe asthma with potentially therapeutic effect on ABPA. Although serum IgE levels are generally higher than $1000 \mathrm{IU} / \mathrm{mL}$ in ABPA patients and the concentration of anti-IgE antibodies required to neutralize this level cannot always be achieved, the efficacy of omalizumab in reducing exacerbations, improving lung function and reducing OCS in ABPA patients has been proved. Efficacy of omalizumab treatment in asthma-ABPA patients has been reported in many case series and case reports [72-78]. The reduction of exacerbations and of total serum IgE levels has generally been demonstrated with a dose of $375 \mathrm{mg}$ every 2 weeks for a period of 1224 months. A reduction of OCS treatment was described in all patients treated. Only one patient in Tillie-Leblond et al. [73] study was not able to reduce OCS dose. Discontinuation of OCS during omalizumab treatment has been described in adult asthmatic patients with ABPA [73,74,77]. A randomized placebo controlled trial conducted by Voskamp et al. [79] on asthma-ABPA patients demonstrated clinical improvement, decreased basophil reactivity to A. fumigatus, decreased basophil FceR1 and surface-bound IgE levels after a 3-month therapy with $750 \mathrm{mg} / \mathrm{monthly}$ of omalizumab. Aside from binding IgE, omalizumab downregulates the cell-surface high affinity IgE receptor FceR1 on basophils. As a result, omalizumab decreases basophil sensitivity and reactivity to A. fumigatus antigens [79].

Also, several case reports on CF-ABPA patients treated with omalizumab described both reduction of the number of exacerbations and improvement in lung function. All patients evaluated in these studies were also able to discontinue OCS [80-86].

These results were not confirmed by the only randomized, doubleblind, placebo-controlled study conducted in order to assess the efficacy of omalizumab as a steroid-sparing agent in CF-ABPA patients. The study was in fact stopped prematurely due to the inability to recruit patients (the total number of patients recruited in the omalizumab group for the blinded phase was 9) and due to a high drop-out: 5 subjects did not continue the study either because of an adverse event $(n=1)$, a lack of efficacy of treatment $(\mathrm{n}=1)$, or unspecified administrative problems. Few side effects have been described in six of nine (66.67\%) subjects in the omalizumab group in the blinded phase, including distal intestinal obstruction syndrome $(n=1)$, infective pulmonary exacerbation of cystic fibrosis $(n=5)$, lower respiratory bacterial tract infection $(n=1)$ and haemoptysis $(\mathrm{n}=1)$ [87].

Since almost a decade, omalizumab has been suggested by Global Initiative for Asthma (GINA) guidelines as an added therapy for severe asthmatic patients, older than 5 years, dependent from inhaled corticosteroid therapy. This treatment may have prevented development of ABPA in asthmatic patients. To our knowledge no studies have been conducted to investigate this correlation.

\section{ABPA Complications}

Recurrent exacerbations, due to either mucoid impaction or worsening of airflow limitation, are the most common complications in ABPA [23]. Untreated ABPA, in addition, can lead to the development of widespread bronchiectasis and chronic pulmonary aspergillosis (CPA).

CPA, consisting in pulmonary and pleural fibrosis and pulmonary cavitations, is responsible for severe complications including pulmonary hypertension and cor pulmonale.

Massive hemoptysis and pneumothorax, potentially life-threatening conditions, can also complicate the course of ABPA, mostly in CFABPA patients [22].

IPA, generally occurring in immunodeficient subjects, has been anecdotically described as an ABPA complication only in patients with prolonged, high-dose corticosteroid therapy. In the event of IPA, $A$. fumigatus infection may disseminate hematogenously to other organs, including the brain, leading to life-threatening conditions such as cerebral infarctions and intracranial hemorrhage [88,89].

Acute hypoxemic respiratory failure, due to large airway collapse because of mucus impaction, represents the most fearsome lifethreatening condition in ABPA. In these patients, therapeutic bronchoscopy (either fiberoptic or rigid) may be indicated, depending on the severity of respiratory failure, to remove the mucoid impaction responsible for proximal collapse [23].

\section{Environmental control by allergen avoidance}

A. fumigatus is ubiquitous. Certain aggravating factors, such as environmental humidity and temperature, influence home fungal growth, while some activities like gardening and cleaning dusty environments increase the exposure to this fungus. Despite ABPA incidence has not been definitively correlated with environmental $A$. fumigatus spore counts, an increase of the latter, when total mold counts are high, has been described. Therefore, it is possible to hypothesize that controlling environmental factors contributing to the growth of $A$. fumigatus can prevent or reduce the antigens exposure to susceptible patients [90].

\section{Conclusions}

Despite advances in our understanding of the role of the allergic response in the pathophysiology of ABPA, limited evidence exists on the pathogenesis of the disease. In addition, early diagnosis of the disease is challenged by the absence of consensus regarding diagnostic criteria, staging and cut-off values of serologic assays. This may delay the administration of a therapy that can potentially prevent permanent lung damage. At present, the only way to allow for an early diagnosis is to routinely investigate all asthmatic and cystic fibrosis patients for ABPA. Future controlled studies are needed to confirm new diagnostic and staging criteria and to standardize cut-off values of various investigations, while the promising role of novel assays (such 
as recombinant Aspergillus antigens and CCL17) need to be confirmed.

In addition, because present primary therapies are mostly based on clinical experience and limited by significant side-effects, it is important to standardize current therapy, while conducting trials on novel immuno-modulatory therapies, including monoclonal antibodies directed against Th2 cytokines.

\section{Disclosures}

The authors declare no conflicts of interest and financial disclosures.

\section{Acknowledgment}

The authors would like to acknowledge Russ Manitt and Vittorio Maglione for the technical support.

\section{References}

1. Fukutomi $Y$, Taniguchi $M$ (2015) Sensitization to fungal allergens: resolved and unresolved issues. Allergol Int 64: 321-331.

2. Hinson KFW, Moon AJ, Plummer NS (1952) Broncho-pulmonary aspergillosis: A review and a report of eight new cases. Thorax 7: 317-333.

3. Eppinger TM, Greenberger PA, White DA, Brown AE, Cunningham-Rundles C (1999) Sensitization to Aspergillus species in the congenital neutrophil disorders chronic granulomatous disease and hyper-lgE syndrome. J Allergy Clin Immunol 104: 1265-1272.

4. Tillie-Leblond I, Tonnel AB (2005) Allergic pulmonary aspergillosis. Allergy 60 1004-1013.

5. Chakrabarti A, Sethi S, Raman DS, Behera D (2002) Eight year study of allergic bronchopulmonary aspergillosis in an Indian teaching hospital. Mycoses 45: 295-299.

6. Denning DW, Pleuvry A, Cole DC (2013) Global burden of allergic bronchopulmonary aspergillosis with asthma and its complication chronic pulmonary aspergillosis in adults. Med Mycol 51: 361-370.

7. Maturu V, Agarwal R (2015) Prevalence of Aspergillus sensitization and allergic bronchopulmonary aspergillosis in cystic fibrosis: systematic review and meta-analysis. Clin Exp Allergy 45: 1765-1778.

8. Geller DE, Kaplowitz H, Light MJ, Colin AA (1999) Allergic Bronchopulmonary Aspergillosis in Cystic Fibrosis. Reported Prevalence, Regional Distribution, and Patient Characteristics. Scientific Advisory Group, Investigators, and Coordinators of the Epidemiologic Study of Cystic Fibrosis. Chest 116: 639-646.

9. Mastella G, Rainisio M, Harms HK, Hodson ME, Koch C, et al. (2000) Allergic bronchopulmonary aspergillosis in cystic fibrosis. A European epidemiological study. Epidemiologic registry of Cystic Fibrosis. Eur Respir J 16: 464-471.

10. Greenberger PA (2002) Allergic Bronchopulmonary Aspergillosis. J Allergy Clin Immunol 110: 685-692.

11. Tanner NT, Judson MA (2008) Diagnosis and treatment of allergic bronchopulmonary aspergillosis. Curr Fungal Infect Rep 2: 199-205.

12. Eickmeier O, Rieber N, Eckrich J, Hector A, Graeppler-Mainka U, et al. (2013) Immune response, diagnosis and treatment of allergic bronchopulmonary aspergillosis in cystic fibrosis lung disease. Curr Pharm Desn19: 3669-3678.

13. Chauhan B, Santiago L, Hutcheson PS, Schwartz HJ, Spitznagel E, et al (2000) Evidence for the involvement of two different MHC class II regions in susceptibility or protection in allergic bronchopulmonary aspergillosis. $J$ Allergy Clin Immunol 106: 723-729.

14. Knutsen AP, Slavin RG (2011) Allergic bronchopulmonary aspergillosis in asthma and cystic fibrosis. Clin Dev Immunol: 2011.

15. Knutsen AP, Kariuki B, Consolino JD, Warrier MR (2006) IL 4 alpha chain receptor (IL4Ralpha) polymorphism in allergic bronchopulmonary aspergillosis. Clin Mol Allergy 4: 3.

16. Brouard J, Knauer N, Boelle PY, Corvol H, Henrion-Caude A, et al. (2005) Influence of interleukin-10 on Aspergillus fumigatus infection in patient with cystic fibrosis. J Infect Dis 191: 1988-1991.

17. Saxena S, Madan T, Shah A, Muralidhar K, Sarma PU (2003) Association of polymorphism in the collagen region of SP-A2 with increased levels of total $\mathrm{IgE}$ antibodies and eosinophilia in patients with allergic bronchopulmonary aspergillosis. J Allergy Clin Immunol 111: 1001-1007.
18. Marchand E, Verellen-Dumoulin C, Mairesse M, Delaunois L, Brancaleone $\mathrm{P}$ et al. (2001) Frequency of cystic fibrosis transmembrane conductance regulato gene mutations and $5 \mathrm{~T}$ allele in patients with allergic bronchopulmonary aspergillosis. Chest 119: 762-767.

19. Lebecque $P$, Pepermans $X$, Marchand E, Leonard A, Leal T (2011) ABPA in adulthood: a CFTR-related disorder. Thorax 66: 540-541.

20. Patterson K, Strek ME (2010) Allergic bronchopulmonary aspergillosis. Proc Am Thorac Soc 7: 237-244.

21. Ritz N, Ammann RA, Casaulta Aebischer C, Schoeni-Affolter F, Schoen $\mathrm{MH}$ (2005) Risk factors for allergic bronchopulmonary aspergillosis and sensitisation to Aspergillus fumigatus in patients with cystic fibrosis. Eur $J$ Pediatr 164: 577-582.

22. Stevens DA, Moss RB, Kurup VP, Knutsen AP, Greenberger P, et al. (2003) Allergic bronchopulmonary aspergillosis in cystic fibrosis-State of the art: Cystic Fibrosis Foundation Consensus Conference. Clin Infect Dis 37: S225-S264

23. Agarwal R, Chakrabarti A, Shah A, Gupta D, Meis JF, et al. (2013) Allergic bronchopulmonary aspergillosis: review of literature and proposal of new diagnostic and classification criteria. Clin Exp Allergy 43: 850-873.

24. Sutrave H, Ward A, Smyth AR, Bhatt J (2012) Pneumomediastinum as presenting feature of allergic bronchopulmonary aspergillosis in a child with cystic fibrosis. J R Soc Med 105: S36-S39.

25. Rosenberg M, Patterson R, Mintzer R, Cooper BJ, Roberts M, et al. (1977) Clinical and immunologic criteria for the diagnosis of allergic bronchopulmonary aspergillosis. Ann Intern Med 86: 405-414.

26. Patterson R, Greenberger PA, Radin RC, Roberts M (1982) Allergic bronchopulmonary aspergillosis: staging as an aid to management. Ann Intern Med 96: 286-291.

27. Patterson R, Greenberger PA, Halwig M, Liotta JL, Roberts M (1986) Allergic bronchopulmonary aspergillosis: natural history and classification of early diagnosis by serologic and roentgenographic studies. Arch Intern Med 146: 916-918.

28. SchwartzHJ, GreenbergerPA(1991) The prevalence of allergic bronchopulmonary aspergillosis in patients with asthma, determined by serologic and radiologic criteria in patients at risk. J Lab Clin Med 117: 138-142.

29. Agarwal R, Maskey D, Aggarwal AN, Saikia B, Garg M, et al. (2013) Diagnostic performance of various tests and criteria employed in allergic bronchopulmonary aspergillosis: a latent class analysis. PLoS One 8: e61105.

30. Agarwal R (2009) Allergic bronchopulmonary aspergillosis. Chest 135: 805-826.

31. Agarwal R, Gupta D, Aggarwal AN, Behera D, Jindal SK (2006) Allergic bronchopulmonary aspergillosis: lessons from 126 patients attending a chest clinic in north India. Chest 130: 442-448.

32. Nikolaizik WH, Weichel M, Blaser K, Crameri R (2002) Intracutaneous tests with recombinant allergens in cystic fibrosis patients with allergic bronchopulmonary aspergillosis and Aspergillus allergy. Am J Respir Crit Care Med 165: 916-921.

33. Fricker-Hidalgo H, Coltey B, Llerena C, Renversez JC, Grillot R, et al. (2010) Recombinant allergens combined with biological markers in the diagnosis of allergic bronchopulmonary aspergillosis in cystic fibrosis patients. Clin Vaccine Immunol 17: 1330-1336.

34. Mirković B, Lavelle GM, Azim AA, Helma K, Gargoum FS, et al. (2015) The basophil surface marker CD203c identifies Aspergillus species sensitization in patients with cystic fibrosis. J Allergy Clin Immunol 137: 436-443.

35. Latzin P, Hartl D, Regamey N, Frey U, Schoeni MH, et al. (2008) Comparison of serum markers for allergic bronchopulmonary aspergillosis in cystic fibrosis. Eur Respir J 31: 36-42.

36. Hartl D, Latzin P, Zissel G, Krane M, Krauss-Etschmann S, et al. (2006) Chemokines indicate allergic bronchopulmonary aspergillosis in patients with cystic fibrosis. Am J Respir Crit Care Med 173: 1370-1376.

37. Agarwal R, Aggarwal AN, Sehgal IS, Dhooria S, Behera D, et al (2015) Performance of serum galactomannan in patients with allergic bronchopulmonary aspergillosis. Mycoses 58: 408-412.

38. Neeld DA, Goodman LR, Gurney JW, Greenberger PA, Fink JN (1990) Computerized tomography in the evaluation of allergic bronchopulmonary aspergillosis. Am Rev Respir Dis 142: 1200-1205. 
39. Mintzer RA, Rogers LF, Kruglik GD, Rosenberg M, Neiman HL, et al. (1978) The spectrum of radiologic findings in allergic bronchopulmonary aspergillosis. Radiology 127: 301-307.

40. Logan PM, Muller NL (1996) High-attenuation mucous plugging in allergic bronchopulmonary aspergillosis. Can Assoc Radiol J 47: 374-377.

41. Ward S, Heyneman L, Lee MJ, Leung AN, Hansell DM (1999) Accuracy of $\mathrm{CT}$ in the diagnosis of allergic bronchopulmonary aspergillosis in asthmatic patients. AJR Am J Roentgenol 173: 937-942.

42. Tamura A, Hebisawa A, Kurashima A, Kawabe Y, Machida K, et al. (1997) The use of bronchofiberscopy for diagnosis of allergic bronchopulmonary aspergillosis. Intern Med 36: 865-869.

43. Cockrill BA, Hales CA (1999) Allergic bronchopulmonary aspergillosis. Annu Rev Med 50: 303-316.

44. Bosken CH, Myers JL, Greenberger PA, Katzenstein AL (1988) Pathologic features of allergic bronchopulmonary aspergillosis. Am J Surg Pathol 12: 216-222.

45. Riscili BP, Wood KL (2009) Noninvasive pulmonary Aspergillus infections. Clin Chest Med 30: 315-335.

46. Walsh TJ, Anaissie EJ, Denning DW, Herbrecht R, Kontoyiannis DP, et al (2008) Treatment of aspergillosis: clinical practice guidelines of the Infectious Diseases Society of America. Clin Infect Dis 46: 327-360.

47. Agarwal R, Aggarwal AN, Dhooria S, Singh Sehgal I, Garg M, et al. (2016) A randomised trial of glucocorticoids in acute-stage allergic bronchopulmonary aspergillosis complicating asthma. Eur Respir J 47: 490-498.

48. Knutsen A, Slavin RG (1992) Allergic bronchopulmonary mycosis complicating cystic fibrosis. Semin Respir Infect 7: 179-192.

49. Ganassini A, Cazzadori A (1995) Invasive pulmonary aspergillosis complicating allergic bronchopulmonary aspergillosis. Respir Med 89: 143-145.

50. Agarwal R (2012) What is the current place of azoles in allergic bronchopulmonary aspergillosis and severe asthma with fungal sensitization. Expert Rev Respir Med 6: 363-371

51. Sinha A, Bagga A (2008) Pulse steroid therapy. Indian J Pediatr 75: $1057-$ 1066.

52. Singh Sehgal I, Agarwal R (2014) Pulse methylprednisolone in allergic bronchopulmonary aspergillosis exacerbations. Eur Respir Rev 23: 149-152.

53. Cohen-Cymberknoh M, Blau H, Shoseyov D, Mei-Zahav M, Efrati O, et al. (2009) Intravenous monthly pulse methylprednisolone treatment for ABPA in patients with cystic fibrosis. J Cyst Fibros 8: 253-257.

54. Agarwal R, Khan A, Aggarwal AN, Saikia B, Gupta D, et al. (2011) Role of inhaled corticosteroids in the management of serological allergic bronchopulmonary aspergillosis (ABPA). Intern Med 50: 855-860.

55. Moreira AS, Silva D, Ferreira AR, Delgado $L$ (2014) Antifungal treatment in allergic bronchopulmonary aspergillosis with and without cystic fibrosis: a systematic review. Clin Exp Allergy 44: 1210-1227.

56. Currie DC, Lueck C, Milburn HJ, Harvey C, Longbottom JL, et al. (1990) Controlled trial of natamycin in the treatment of allergic bronchopulmonary aspergillosis. Thorax 45: 447-450.

57. Sehgal IS, Agarwal R (2014) Role of inhaled amphotericin in allergic bronchopulmonary aspergillosis. J Postgrad med 60: 41-45.

58. Shale DJ, Faux JA, Lane DJ (1987) Trial of ketoconazole in non-invasive pulmonary aspergillosis. Thorax 42: 26-31.

59. Stevens DA, Schwartz HJ, Lee JY, Moskovitz BL, Jerome DC, et al. (2000) A randomized trial of itraconazole in allergic bronchopulmonary aspergillosis. $\mathrm{N}$ Engl J Med 342: 756-762.

60. Wark PA, Hensley MJ, Saltos N, Boyle MJ, Toneguzzi RC, et al. (2003) Anti-inflammatory effect of itraconazole in stable allergic bronchopulmonary aspergillosis: a randomized controlled trial. J Allergy Clin Immunol 111: 952-957.

61. Elphick HE, Southern KW (2014) Antifungal therapies for allergic bronchopulmonary aspergillosis in people with cystic fibrosis. Cochrane Database Syst Rev 11: CD002204.

62. Hardin T, Graybill JR, Fetchick R, Woestenborghs R, Rinaldi MG, et al. (1988) Pharmacokinetics of itraconazole following oral administration in normal volunteers. Antimicrob Agents Chemother 32: 1310-1313.
63. Pasqualotto AC, Denning DW (2007) Generic substitution of itraconazole resulting in sub-therapeutic levels and resistance. Int J Antimicrob Agents 30: 93-94.

64. Hope WW, Billaud EM, Lestner J, Denning DW (2008) Therapeutic drug monitoring for triazoles. Curr Opin Infect Dis 21: 580-586.

65. Varis T, Kaukonen KM, Kivistö KT, Neuvonen PJ (1998) Plasma concentrations and effects of oral methylprednisolone are considerably increased by itraconazole. Clin Pharmacol Ther 64: 363-368.

66. Maturu VN, Agarwal R (2015) Acute Invasive Pulmonary Aspergillosis Complicating Allergic Bronchopulmonary Aspergillosis: Case Report and Systematic Review. Mycopathologia 180: 209-215.

67. Glackin L, Leen G, Elnazir B, Greally P (2009) Voriconazole in the treatment of allergic bronchopulmonary aspergillosis in cystic fibrosis. Ir Med J 102: 29.

68. Chishimba L, Niven RM, Cooley J, Denning DW (2012) Voriconazole and posaconazole improve asthma severity in allergic bronchopulmonary aspergillosis and severe asthma with fungal sensitization. J Asthma 49: 423-33.

69. Moss RB (2014) Treatment options in severe fungal asthma and allergic bronchopulmonary aspergillosis. Eur Respir J 43: 1487-1500.

70. Bains SN, Judson MA (2012) Allergic bronchopulmonary aspergillosis. Clin Chest Med 33: 265-281.

71. Agarwal R, Aggarwal AN, Sehgal IS, Dhooria S, Behera D, et al (2016) Utility of $\lg E$ (total and Aspergillus fumigatus specific) in monitoring for response and exacerbations in allergic bronchopulmonary aspergillosis. Mycoses 59: 1-6.

72. Sastre I, Blanco J, Mata H, García F (2012) A case of allergic bronchopulmonary aspergillosis treated with omalizumab. J Investig Allergol Clin Immunol 22 145-147.

73. Tillie-Leblond I, Germaud P, Leroyer C, Tétu L, Girard F, et al. (2011) Allergic bronchopulmonary aspergillosis and omalizumab. Allergy 66: 1254-1256.

74. Evans MO, Morris MJ, Coop CA, Evans SE (2015) Omalizumab, an additiona therapy for allergic bronchopulmonary aspergillosis. Ann Allergy Asthma Immunol 115: 250-251.

75. Homma T, Kurokawa M, Matsukura S, Yamaguchi M, Adachi M (2013) AntiIgE therapy for allergic bronchopulmonary aspergillosis. J Microbiol Immuno Infect 49: 459-63.

76. Collins J, Devos G, Hudes G, Rosenstreich D (2012) Allergic bronchopulmonary aspergillosis treated successfully for one year with omalizumab. J Asthma Allergy 5: 65-70.

77. Lin RY, Sethi S, Bhargave GA (2010) Measured immunoglobulin E in allergic bronchopulmonary aspergillosis treated with omalizumab. J Asthma 47: 942-945.

78. Beam KT, Coop CA (2015) Steroid sparing effect of omalizumab in seropositive allergic bronchopulmonary aspergillosis. Allergy Rhinol (Providence) 6: 143-145.

79. Voskamp AL, Gillman A, Symons K, Sandrini A, Rolland JM, et al. (2015 Clinical efficacy and immunologic effects of omalizumab in allergic bronchopulmonary aspergillosis. J Allergy Clin Immunol Pract 3: 192-199.

80. Wong R, Wong M, Robinson PD, Fitzgerald DA (2013) Omalizumab in the management of steroid dependent allergic bronchopulmonary aspergillosis (ABPA) complicating cystic fibrosis. Paediatr Respir Rev 14: 22-24.

81. Elmallah MK, Hendeles L, Hamilton RG, Capen C, Schuler PM (2012) Management of patients with cystic fibrosis and allergic bronchopulmonary aspergillosis using anti-immunoglobulin e therapy (omalizumab). J Pediatr Pharmacol Ther 17: 88-92.

82. Zicari AM, Celani C, De Castro G, Valerio De Biase R, Duse M (2014) Anti IgE antibody as treatment of allergic bronchopulmonary aspergillosis in a patient with cystic fibrosis. Eur Rev Med Pharmacol Sci 18: 1839-1841.

83. Lebecque $P$, Leonard $A$, Pilette $C$ (2009) Omalizumab for treatment of $A B P A$ exacerbations in CF patients. Pediatr Pulmonol 44: 516

84. Zirbes JM, Milla CE (2008) Steroid-sparing effect of omalizumab for allergic bronchopulmonary aspergillosis and cystic fibrosis. Pediatr Pulmonol 43: 607-610.

85. van der Ent CK, Hoekstra H, Rijkers GT (2007) Successful treatment of allergic bronchopulmonary aspergillosis with recombinant anti-lgE antibody. Thorax 62: 276-277.

86. Kanu A, Patel K (2008) Treatment of allergic bronchopulmonary aspergillosis (ABPA) in CF with anti-IgE antibody (omalizumab). Pediatr Pulmonol 43: 1249-1251. 
Citation: Leonardi L, Cinicola BL, Laitano R, Duse M (2016) Allergic Bronchopulmonary Aspergillosis: Diagnostic and Treatment Challenges. J Pulm Respir Med 6: 361. doi: 10.4172/2161-105X.1000361

87. Jat KR, Walia DK, Khairwa A (2015) Anti-lgE therapy for allergic bronchopulmonary aspergillosis in people with cystic fibrosis. Cochrane Database Syst Rev: CD010288.

88. Denning DW (1998) Invasive aspergillosis. Clin Infect Dis 26: 781-803.
89. Kousha M, Tadi R, Soubani AO (2011) Pulmonary aspergillosis: A clinical review. Eur Respir rev 20: 156-174.

90. Sharpe R, Thornton CR, Osborne NJ (2014) Modifiable factors governing indoor fungal diversity and risk of asthma. Clin Exp Allergy 44: 631-641.
Citation: Leonardi L, Cinicola BL, Laitano R, Duse M (2016) Allergic Bronchopulmonary Aspergillosis: Diagnostic and Treatment Challenges. J Pulm Respir Med 6: 361. doi: 10.4172/2161-105X.1000361
OMICS International: Publication Benefits \& Features

Unique features:

- Increased global visibility of articles through worldwide distribution and indexing

- Showcasing recent research output in a timely and updated manner

Special issues on the current trends of scientific research

Special features:

- $700+$ Open Access Journals

$50,000+$ Editorial team

Rapid review process

- Quality and quick editorial, review and publication processing

- Indexing at major indexing services

- Sharing Option: Social Networking Enabled

- Authors, Reviewers and Editors rewarded with online Scientific Credits

- Befter discount for your subsequent articles

Submit your manuscript at: www.omicsonline.org/submission/ 